\title{
Geometry and dynamics of vortex sheets in 3 dimensions
}

\author{
D.A. Burton* R.W. Tucker ${ }^{\dagger}$
}

\begin{abstract}
We consider the properties and dynamics of vortex sheets from a geometrical, coordinate-free, perspective. Distribution-valued forms (de Rham currents) are used to represent the fluid velocity and vorticity due to the vortex sheets. The smooth velocities on either side of the sheets are solved in terms of the sheet strengths using the language of double forms. The classical results regarding the continuity of the sheet normal component of the velocity and the conservation of vorticity are exposed in this setting. The formalism is then applied to the case of the self-induced velocity of an isolated vortex sheet. We develop a simplified expression for the sheet velocity in terms of representative curves. Its relevance to the classical Localized Induction Approximation (LIA) to vortex filament dynamics is discussed.
\end{abstract}

\section{Introduction}

\subsection{Notation and conventions}

Let $\mathcal{M}$ be a $C^{\infty}$ manifold and let $c:[0,1]^{p} \rightarrow \mathcal{M}$ be an oriented $p$-chain on $\mathcal{M}$. The image of $c$ shall be labelled $\mathcal{D}_{c}$ and $b \mathcal{D}_{c} \equiv \mathcal{D}_{\partial c}$. Distributions (de Rham currents) on the space of test forms on $\mathcal{M}$ shall be distinguished by a $D$ subscript. Our attention will be focussed on tensors on subsets of $\mathbb{R}^{3}$, with $g$ and $\nabla$ being the standard metric and Levi-Civita connection on $\mathbb{R}^{3}$

*Department of Physics, Lancaster University, UK (e-mail: d.burton@lancaster.ac.uk)

${ }^{\dagger}$ Department of Physics, Lancaster University, UK (e-mail: r.tucker@lancaster.ac.uk) 
respectively. The metric dual of a vector field $X$, more precisely $g(X,-)$, is written $\tilde{X}$. Similarly, the metric dual of a 1 -form $\alpha, g^{-1}(\alpha,-)$, is written $\tilde{\alpha}$. The length of $X$ (respectively $\alpha$ ), written $|X|$ (respectively $|\alpha|$ ), is equal to $\sqrt{g(X, X)}$ (respectively $\sqrt{g^{-1}(\alpha, \alpha)}$ ). Furthermore, $\star$ is the Hodge map associated with $g$. We may indicate the degree of a homogenous form, homogenous chain or homogenous distribution by writing it underneath the form, chain or distribution. The interior, or contraction, operation on a form $\alpha$, taken with respect to the vector field $X$, is denoted $\iota_{X} \alpha$. Most tensor fields, chains and distributions in this article are implicitly one-parameter families with $t$, the Newtonian time, as the parameter.

\section{Vorticity and the Euler equations}

Before turning to the main focus of this article, we recall a few concepts regarding classical fluid flows. Consider a Newtonian, inviscid and incompressible fluid modelled by a smooth flow velocity vector $V$ on an open bounded region $\mathcal{D}_{\Omega} \subset \mathbb{R}^{3}$ described by the 3 -chain $\Omega$. That the fluid is incompressible means that

$$
d \star \tilde{V}=0 .
$$

That the fluid is Newtonian and inviscid means that $V$ satisfies the Euler equation of motion (see, for example, $[1,3,7,10]$ )

$$
\partial_{t} \tilde{V}+\nabla_{V} \tilde{V}=-d p
$$

where $p$ is the fluid pressure. The fluid density is assumed to be constant, and so has been normalised to unity. The archetypal boundary condition for such a flow is the no-through-flow condition,

$$
\left.g(N, V)\right|_{b \mathcal{D}_{\Omega}}=0
$$

where $N$ is normal to $b \mathcal{D}_{\Omega}$. In terms of the Hodge map $\star$ this can be written

$$
\partial \Omega^{*} \star \tilde{V}=0
$$

The vorticity 2-form of $V$ is

$$
\omega \equiv d \tilde{V}
$$

One can show that

$$
\nabla_{V} \tilde{V}=\mathcal{L}_{V} \tilde{V}-\frac{1}{2} d|V|^{2}
$$


and so acting with the exterior derivative on (2) yields

$$
\partial_{t} \omega+\mathcal{L}_{V} \omega=0 .
$$

A 2-chain $\Sigma$ that comoves with the fluid satisfies

$$
\frac{d}{d t} \int_{\Sigma} \alpha=\int_{\Sigma}\left(\partial_{t} \alpha+\mathcal{L}_{V} \alpha\right)
$$

for any differential form $\alpha$. Therefore, for any comoving $\Sigma$

$$
\frac{d}{d t} \int_{\Sigma} \omega=0
$$

and so the vortex strength $\Gamma[\Sigma]$ in $\Sigma$,

$$
\begin{aligned}
\Gamma[\Sigma] & \equiv \int_{\Sigma} \omega \\
& =\int_{\partial \Sigma} \tilde{V}
\end{aligned}
$$

is a conserved quantity. This is one of the Helmholtz vortex theorems $[1,3$, $7,10]$.

\section{On modelling vortex sheets}

In many practical cases the vorticity is concentrated in certain regions of $\mathcal{D}_{\Omega}$. In what follows we are concerned entirely with vorticity that is confined to thin sheets. One way to proceed is to consider idealized sheets that are arbitrarily thin and model them as 2-dimensional submanifolds of $\mathcal{D}_{\Omega}$. These submanifolds partition $\mathcal{D}_{\Omega}$ into distinct regions, each of which has a smooth fluid velocity vector defined on it. The fact that the flow velocity vector is chosen to be discontinuous across the submanifolds leads to a non-zero fluid vorticity. The fluid velocities in each region will be related by a junction condition across the submanifolds obtained via a distribution-valued flow velocity 1 -form $\tilde{V}_{D}$ in $\mathcal{M} \supset \mathcal{D}_{\Omega}$ that satisfies

$$
d \star \tilde{V}_{D}=0 .
$$

Using a generalization of Green's identities [5] that apply to differential forms we express the fluid velocity vector in terms of the velocity discontinuities. The distribution-valued vorticity 2 -form $\omega_{D}$ given by

$$
\omega_{D}=d \tilde{V}_{D}
$$


is shown to satisfy

$$
\partial_{t} \omega_{D}+\mathcal{L}_{U} \omega_{D}=0
$$

over test forms on $\mathcal{D}_{\Omega}$, if the pressure is continuous across the sheets and where $U$ is the sheet velocity. We conclude this article by presenting a simple, but illuminating, approximation to the fluid velocity vector due to a vortex sheet in an unbounded flow.

\section{The self-induced velocity of a set of vortex sheets}

\section{1 de Rham's unification of forms and chains}

The objects in de Rham's unified description of differential forms and chains [6] are known as currents. A current (or distribution-valued form) $T_{D}$ on $\mathcal{M}$ is a linear functional $T_{D}[\cdot]$ defined on the vector space of all $C^{\infty}$ forms $\phi$ with compact support (also known as test forms). If $T_{D}[\phi]=0$ for each homogenous test form which is not of degree $p$ then the degree of $T_{D}$ is $n-p$. Canonical examples of currents stem from homogenous $p$-chains,

$$
c_{D}[\phi]=\left\{\begin{array}{lll}
\int_{(p)} \underset{(q)}{ }{ }_{(q)} & : & p=q, \\
0 & : & p \neq q .
\end{array}\right.
$$

and locally integrable homogenous forms,

$$
\beta_{D}[\phi]=\left\{\begin{array}{lll}
\int_{\mathcal{M}} \underset{(p)}{\beta \wedge} \underset{(q)}{\phi}: & q=n-p \\
0 & : & q \neq n-p .
\end{array}\right.
$$

Note that if $c$ is a $p$-chain then $c_{D}$ is an $(n-p)$-current, but that if $\beta$ is a $p$-form then $\beta_{D}$ is a $p$-current.

The exterior product of a current $T_{D}$ and a $C^{\infty}$ form $\alpha$ is defined by

$$
T_{D} \wedge \alpha[\phi] \equiv T_{D}[\alpha \wedge \phi]
$$

and also satisfies

$$
\underset{(p)}{\alpha} \wedge \underset{(q)}{T_{D}} \equiv(-1)^{p q} T_{D} \wedge \alpha
$$


for a $p$-form $\alpha$ and $q$-current $T_{D}$. The boundary $\partial T_{D}$ of a current $T_{D}$ is defined by

$$
\partial T_{D}[\phi] \equiv T_{D}[d \phi]
$$

and the exterior derivative $d T_{D}$ of $T_{D}$ is

$$
d T_{D} \equiv\left(\partial T_{D}\right)^{\eta}
$$

where

$$
\underset{(p)}{T_{D}{ }^{\eta}} \equiv(-1)^{p} T_{D}
$$

Likewise, the boundary $\partial \alpha$ of a $C^{\infty}$ form $\alpha$ is

$$
\partial \alpha \equiv(d \alpha)^{\eta}
$$

where

$$
\underset{(p)}{\alpha^{\eta}} \equiv(-1)^{p} \alpha
$$

Using (18) and (19) one can show that

$$
d\left(T_{D} \wedge \alpha\right)=d T_{D} \wedge \alpha+T_{D}^{\eta} \wedge d \alpha
$$

or, equivalently,

$$
\partial\left(T_{D} \wedge \alpha\right)=\partial T_{D} \wedge \alpha^{\eta}+T_{D} \wedge \partial \alpha .
$$

The symmetry condition

$$
\underset{(p)}{\alpha} \wedge \underset{(p)}{\wedge}=\underset{(p)}{\beta} \wedge \underset{(p)}{\beta} \underset{,}{\beta}
$$

is used to define the Hodge dual (or adjoint) $\star T_{D}$ of a current $T_{D}$. Specifically, we demand that

$$
\underset{(p)}{T_{D}} \wedge \underset{(p)}{\alpha}=\underset{(p)}{\alpha} \wedge \underset{(p)}{\wedge} T_{D},
$$

and are lead to the definition of $\star T_{D}$

$$
\star T_{D}[\phi] \equiv T_{D}\left[\star \phi^{\mu}\right]
$$

where

$$
\underset{(p)}{\alpha^{\mu}} \equiv(-1)^{p(n-p)} \alpha .
$$


Using (27) one can show that

$$
\left.\star \underset{(0)}{\left(T_{D}\right.} \wedge \alpha\right)=\underset{(0)}{T_{D}} \wedge \star \alpha .
$$

The interior operator $\iota_{X}$ is defined on currents to be

$$
\iota_{X} T_{D}[\phi] \equiv-T_{D}^{\eta}\left[\iota_{X} \phi\right]
$$

where $X$ is a smooth vector field. Equations (18), (19), (30) and the identity

$$
\mathcal{L}_{X} \alpha=\iota_{X} d \alpha+d \iota_{X} \alpha
$$

are used to obtain the Lie derivative of $T_{D}$

$$
\mathcal{L}_{X} T_{D}[\phi] \equiv-T_{D}\left[\mathcal{L}_{X} \phi\right]
$$

Furthermore, the partial derivative $\partial_{t} T_{D}$ of a current $T_{D}$ is

$$
\partial_{t} T_{D}[\phi] \equiv \frac{d}{d t}\left(T_{D}[\phi]\right)-T_{D}\left[\partial_{t} \phi\right]
$$

\subsection{Fluid flow velocity as a current}

As we mentioned in the introduction, in order to model the dynamics of an arbitrarily thin sheet of vorticity we represent the fluid in terms of distribution-valued forms, i.e. currents. More specifically, we consider currents over test forms with compact support in $\mathcal{M} \subset \mathbb{R}^{3}$ where $\mathcal{M} \supset \mathcal{D}_{\Omega}$. Let $\mathcal{D}_{\Omega}$ be partitioned into the open sets $\left\{\mathcal{D}_{\Omega(1)}, \mathcal{D}_{\Omega(2)}, \ldots, \mathcal{D}_{\Omega(N)}\right\}, N \in \mathbb{Z}^{+}$, by a set of 2-chains that will represent the vortex sheets (see figure 1). Then, for each $j \in I \subset \mathbb{Z}^{+}$let $V_{(j)}$ be a smooth vector field defined over $\mathcal{D}_{\Omega(j)}$ with the properties

$$
\begin{gathered}
d \tilde{V}_{(j)}=0, \\
d \star \tilde{V}_{(j)}=0 .
\end{gathered}
$$

Consider the current $\tilde{V}_{D}$ with compact support in $\mathcal{M}$ given by

$$
\tilde{V}_{D}=\sum_{j \in I} \Omega_{D(j)} \wedge \tilde{V}_{(j)}
$$




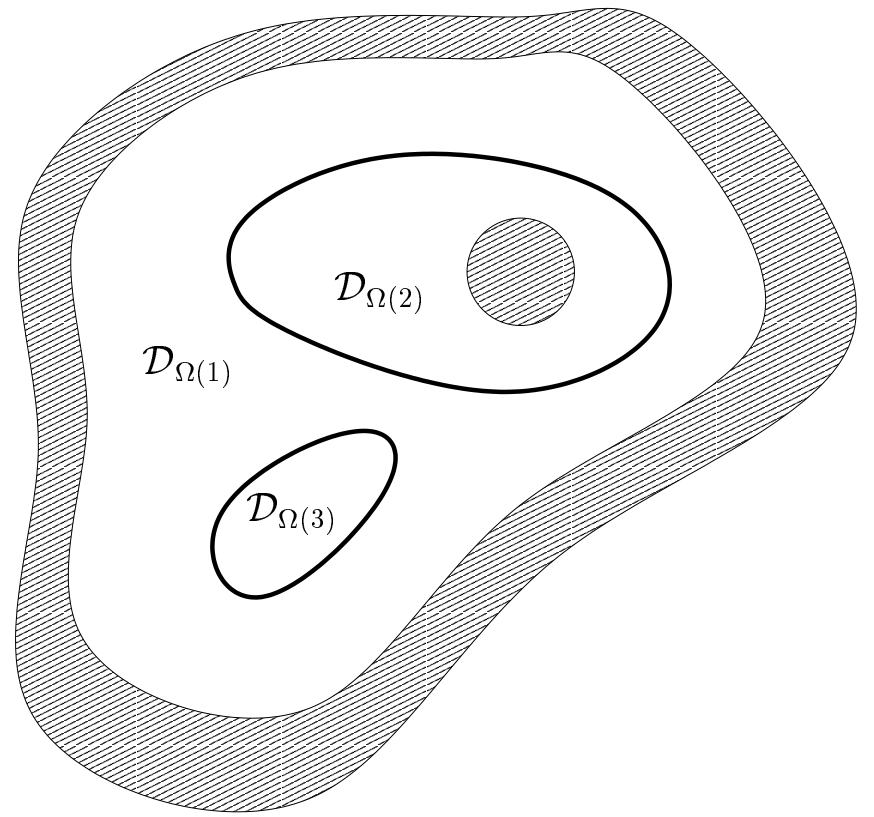

Figure 1: The bounded region $\mathcal{D}_{\Omega}$ has been partitioned into $\left\{\mathcal{D}_{\Omega(1)}, \mathcal{D}_{\Omega(2)}, \mathcal{D}_{\Omega(3)}\right\}$. The thick lines indicate vortex sheets, across which the velocity field is discontinuous.

subject to

$$
d \star \tilde{V}_{D}=0
$$

and introduce the vorticity 2-current $\omega_{D}$,

$$
\omega_{D} \equiv d \tilde{V}_{D}
$$

Using (23) and (19) to expand (38) and (37) we find that

$$
\begin{gathered}
\omega_{D}=-\sum_{j \in I} \partial \Omega_{D(j)} \wedge \tilde{V}_{(j)}, \\
\sum_{j \in I} \partial \Omega_{D(j)} \wedge \star \tilde{V}_{(j)}=0 .
\end{gathered}
$$

\section{The junction condition across the sheets}

One can gain more insight into the structure of (39) and (40) by writing $\left\{\partial \Omega_{(1)}, \ldots, \partial \Omega_{(N)}\right\}$ in terms of their component 2-cubes. Introduce $S \equiv$ 
$\left\{\sigma_{(1)}, \ldots, \sigma_{(M)}\right\}$, where $M \in \mathbb{Z}^{+}$. Each vortex sheet has two elements of $S$ associated with it. The criterion defining the pair $\left\{\sigma_{(a)}, \sigma_{(b)}\right\}$ is that $\sigma_{(a)}^{*} \alpha=-\sigma_{(b)}^{*} \alpha$ for any $C^{\infty}$ form $\alpha$ on $\mathcal{M}$. Let the elements of $I_{v} \subset \mathbb{Z}^{+}$ index all such pairs, and let those of $I_{s} \subset \mathbb{Z}^{+}$index the remaining elements of $S$ (those that correspond to a solid boundary). Hence, if $n$ is the number of elements of $I_{v}$ and $m$ is the number of elements of $I_{s}$ then $2 n+m=M$. Denote the $k$ th pair as $\left\{\sigma_{(k)}^{+}, \sigma_{(k)}^{-}\right\}$and introduce the 2-cube $\Sigma_{(k)}$ (with image $\left.\mathcal{D}_{\Sigma(k)}\right)$ where

$$
\begin{aligned}
\Sigma_{(k)}^{*} \alpha & =-\sigma_{(k)}^{+*} \alpha \\
& =\sigma_{(k)}^{-*} \alpha
\end{aligned}
$$

for all $C^{\infty}$ forms on $\mathcal{M}$ and for all $k \in I_{v}$. Let $\left\{\mathcal{D}^{+}, \mathcal{D}^{-}\right\}$be the two subsets of $\mathcal{D}_{\Omega}$ partitioned by $\mathcal{D}_{\Sigma(k)}$. Then if $\left\{A^{+}, A^{-}\right\}$are $C^{\infty}$ tensors defined on $\left\{\mathcal{D}^{+}, \mathcal{D}^{-}\right\}$and $x^{+} \in \mathcal{D}^{+}, x^{-} \in \mathcal{D}^{-}$, the discontinuity in $A$ across $\mathcal{D}_{\Sigma(k)}$ is

$$
[A]_{(k)}(x) \equiv \lim _{x^{+} \rightarrow x} A^{+}\left(x^{+}\right)-\lim _{x^{-} \rightarrow x} A^{-}\left(x^{-}\right)
$$

at any point $x \in \mathcal{D}_{\Sigma(k)}$. The orientation of each $\Sigma_{(k)}$ can be chosen such that (39) and (40) can be written in the form

$$
\begin{gathered}
\omega_{D}=\sum_{k \in I_{v}} \Sigma_{D(k)} \wedge[\tilde{V}]_{(k)}-\sum_{a \in I_{s}} \sigma_{D(a)} \wedge \tilde{V}_{b(a)} \\
\sum_{k \in I_{v}} \Sigma_{D(k)} \wedge[\star \tilde{V}]_{(k)}-\sum_{a \in I_{s}} \sigma_{D(a)} \wedge \tilde{V}_{b(a)}=0
\end{gathered}
$$

where each $V_{b(a)}, a \in I_{s}$, is a smooth vector field that coincides with the solid boundary value of the fluid velocity. Equation (43) shows the individual contributions to $\omega_{D}$ due to the vortex sheets (the discontinuities in the fluid velocity) and the presence of the physical boundary.

Equation (44) deserves closer scrutiny. If we act with (44) on test forms that vanish on $\mathcal{D}_{\Sigma(k)}, \forall k \in I_{v}-\{l\}$, and vanish on $b \mathcal{D}_{\Omega}$ we find that

$$
\int_{\Sigma_{(l)}}[\star \tilde{V}]_{(l)} \wedge \phi=0
$$

Since $\Sigma_{(l)}$ annihilates the 1-form $\tilde{N}_{(l)}$,

$$
\Sigma_{(l)}^{*} \tilde{N}_{(l)}=0,
$$


where $N_{(l)}$ is normal to $\Sigma_{(l)}$, it follows that

$$
\int_{\Sigma_{(l)}} \Pi_{N_{(l)}}[\star \tilde{V}]_{(l)} \wedge \phi=0
$$

where the idempotent $\Pi_{X}$ is the $X$-orthogonal projection map

$$
\begin{gathered}
\Pi_{X} \tilde{X}=0 \\
\Pi_{X} \alpha(Y)=\alpha\left(\Pi_{X} Y\right), \\
\Pi_{X}(\alpha \otimes \beta)=\Pi_{X} \alpha \otimes \Pi_{X} \beta .
\end{gathered}
$$

Equation (47) holds for all such $\phi$, most notably for a $\phi$ coinciding with $\# \Pi_{N_{(l)}}[\star \tilde{V}]_{(l)}$ in $\mathcal{D}_{\Sigma(l)}$ where \# is the induced Hodge map on $\mathcal{D}_{\Sigma(l)}$. Thus, we obtain the junction condition

$$
\Pi_{N_{(l)}}[\star \tilde{V}]_{(l)}=0 .
$$

Equation (51) expresses the fact that the normal component of the flow velocity is continuous across each vortex sheet. Similarly, by choosing test forms that vanish on $\mathcal{D}_{\Sigma(k)}, \forall k \in I_{v}$, it can be shown that

$$
\Pi_{N_{b}} \star \tilde{V}_{b}=0
$$

where $N_{b}$ is normal to $b \mathcal{D}_{\Omega}$ and $V_{b}$ is a piecewise smooth vector field satisfying

$$
V_{b}(x)=V_{b(a)}(x)
$$

at $x \in \mathcal{D}_{\sigma(a)}$ for $a \in I_{s}$. Thus the normal component of $V_{b}$ must vanish at the physical boundaries, which is just the traditional no-through-flow boundary condition. Within this formalism one can view the solid boundary as a collection of vortex sheets with zero fluid velocity ${ }^{1}$ outside of $\mathcal{D}_{\Omega}$. The next step is to construct a representation of a particular solution to (34) and (35).

\footnotetext{
${ }^{1}$ This observation is the basis of the boundary-integral methods $[3,7]$ used for constructing flows in bounded regions.
} 


\subsection{Double forms}

Let $\mathcal{M}$ and $\mathcal{N}$ be two manifolds. Then a double form $[5,6]$ on $\mathcal{M} \times \mathcal{N}$ is a differential form on $\mathcal{M}$ with coefficients that are differential forms on $\mathcal{N}$ or, equivalently, a differential form on $\mathcal{N}$ with coefficients that are differential forms on $\mathcal{M}$. Let $\mathcal{M}$ and $\mathcal{N}$ have dimensions $m$ and $n$ and let $x \in \mathcal{M}$ and $y \in \mathcal{N}$ be points with coordinates $\left\{x^{1}, \ldots, x^{m}\right\}$ and $\left\{y^{1}, \ldots, y^{m}\right\}$ in a local chart on $\mathcal{M}$ and in a local chart on $\mathcal{N}$ respectively. Then a degree $(p, q)$ double form $\gamma$ on $\mathcal{M} \times \mathcal{N}$ shall be written locally

$$
\gamma(x, y)=\gamma_{a_{1} \ldots a_{p} \mid b_{1} \ldots b_{q}}(x, y)\left(d x^{a_{1}} \wedge \cdots \wedge d x^{a_{p}}\right) \odot\left(d y^{b_{1}} \wedge \cdots \wedge d y^{b_{q}}\right),
$$

where the Einstein summation convention has been adhered to i.e. repeated labels are implicitly summed over. The symbol $\odot$ indicates a "symmetric" product where

$$
(\alpha \odot \beta) \wedge(A \odot B) \equiv(\alpha \wedge A) \odot(\beta \wedge B)
$$

for forms $\alpha$ and $A$ on $\mathcal{M}$ and $\beta$ and $B$ on $\mathcal{N}$. Hence, if $\alpha$ is a $(p, q)$ double form and $\beta$ is a $\left(p^{\prime}, q^{\prime}\right)$ double form then

$$
\alpha \wedge \beta=(-1)^{\left(p p^{\prime}+q q^{\prime}\right)} \beta \wedge \alpha .
$$

In this article we only need to consider situations when $\mathcal{M}=\mathcal{N}$, and will sometimes refer to forms defined on $\mathcal{M}$, as opposed to $\mathcal{M} \times \mathcal{M}$, as single forms. One can define symmetric double forms, i.e. those that satisfy

$$
\beta(x, y)=\beta(y, x)
$$

for $(x, y) \in \mathcal{M} \times \mathcal{M}$. Given a chain $c$ on $\mathcal{M}$ and a double form $\alpha$ on $\mathcal{M} \times \mathcal{M}$ one can integrate with respect to $x$ over $c$,

$$
\int_{c(x)} \alpha(x, y),
$$

or with respect to $y$ over $c$,

$$
\int_{c(y)} \alpha(x, y),
$$


which will in general yield different single forms on $\mathcal{M}$. For our purposes we only need to consider double forms that can be written as the exterior product of single forms and a symmetric double form. For simplicity of notation, when we integrate such forms we will write

$$
\int_{c} \alpha
$$

where the integration is with respect to $x$ if the single forms are written as functions of $x$ within the integrand. The same rule holds with respect to $y$. For example, if $\alpha$ is a symmetric double form and $\beta$ is a single form then

$$
\int_{c}(\alpha \wedge \beta)(x)=\int_{c(y)} \alpha(x, y) \wedge \beta(y) .
$$

\subsection{The Poisson-Beltrami equation on $\mathbb{R}^{n}$}

The Laplace-Beltrami operator $\triangle \equiv-(d \delta+\delta d)$ gives rise to what we call the Poisson-Beltrami equation,

$$
-\triangle \Phi=\Lambda
$$

where $\Phi$ and $\Lambda$ are, in general, inhomogenous forms on $\mathcal{M} \subset \mathbb{R}^{n}, \operatorname{dim}(\mathcal{M})=$ $n$. Solutions to this equation are given in terms of the fundamental solution $\gamma$ to the Laplace-Beltrami equation [5],

$$
\triangle \gamma=0
$$

where $\gamma$ is the double inhomogenous form on $\mathcal{M} \times \mathcal{M}$

$$
\gamma(x, y)=\sum_{p=0}^{n} f(x, y) \delta_{A \mid B} E_{(p)}^{A}(x) \odot E_{(p)}^{B}(y)
$$

where $\left\{E_{(p)}^{1}, E_{(p)}^{2}, \ldots, E_{(p)}^{q}\right\}, q=n ! /(p !(n-p) !)$, is a $p$-form basis constructed entirely from a Cartesian coframe $\left\{d x^{1}, d x^{2}, \ldots, d x^{n}\right\}$, that is

$$
d x^{a} \wedge \star d x^{b}=\delta^{a b} \star 1 .
$$




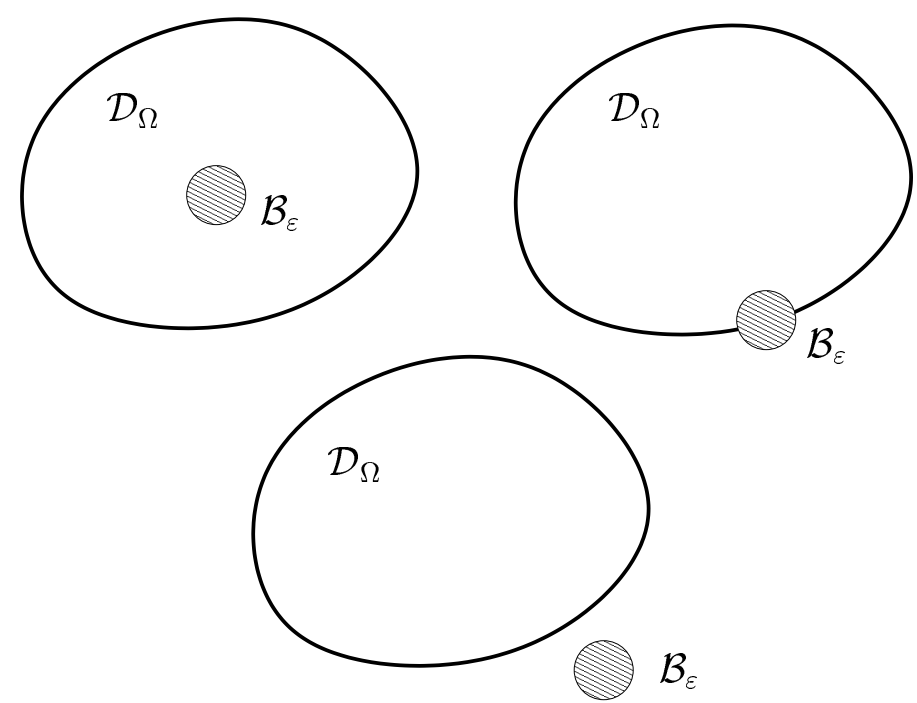

Figure 2: There are three distinct cases to consider when constructing the solution to the Poisson-Beltrami equation. In each case the point at which the solution is to be calculated is at the centre of the open $n$-ball $\mathcal{B}_{\varepsilon}$. The thick line indicates the image $\mathcal{D}_{\Upsilon \varepsilon}=b \mathcal{D}_{\Omega}-\mathcal{B}_{\varepsilon}$ of the one-parameter family of $(n-1)$-chains $\Upsilon_{\varepsilon}$.

The double scalar $f$ is

$$
f(x, y)=\left\{\begin{array}{lll}
\frac{1}{(n-2) v_{n-1}} \frac{1}{r(x, y)^{n-2}} & : & n>2 \\
\frac{1}{2 \pi} \ln \frac{1}{r(x, y)} & : & n=2
\end{array}\right.
$$

where $v_{n-1}$ is the volume of the unit $(n-1)$-sphere in $\mathbb{R}^{n}$ and

$$
r(x, y) \equiv \sqrt{\delta_{a b}\left(x^{a}-y^{a}\right)\left(x^{b}-y^{b}\right)} .
$$

Let $\Omega$ be an $n$-chain that describes the open set $\mathcal{D}_{\Omega} \subset \mathcal{M}$. Furthermore, let $\mathcal{B}_{\varepsilon} \subset \mathcal{M}$ be a one-parameter family of open $n$-balls of radius $\varepsilon>0$ whose centres are at the same point $x \in \mathcal{M}$ for all $\varepsilon$. There are three distinct possibilities regarding the location of $x$ (see figure 2). The point $x$ is either inside $\mathcal{D}_{\Omega}$, outside $\overline{\mathcal{D}}_{\Omega}$, or in $b \mathcal{D}_{\Omega}$. Let $\Omega_{\varepsilon}$ and $\Upsilon_{\varepsilon}$ be chains that describe $\mathcal{D}_{\Omega \varepsilon}=\mathcal{D}_{\Omega}-\mathcal{B}_{\varepsilon}$ and $\mathcal{D}_{\Upsilon \varepsilon}=b \mathcal{D}_{\Omega}-\mathcal{B}_{\varepsilon}$ respectively. One can show that a 
particular solution of (62) satisfies [5] :

$$
\Psi(x)=\left\{\begin{array}{lll}
0 & : & x \notin \overline{\mathcal{D}}_{\Omega}, \\
\frac{1}{2} \Phi(x) & : & x \in b \mathcal{D}_{\Omega}, \\
\Phi(x) & : & x \in \mathcal{D}_{\Omega}
\end{array}\right.
$$

where

$$
\begin{aligned}
\Psi(x) & \equiv \lim _{\varepsilon \rightarrow 0} \int_{\Omega_{\varepsilon}} \Lambda \wedge \star \gamma(x) \\
& -\lim _{\varepsilon \rightarrow 0} \int_{\Upsilon_{\varepsilon}}(\delta \Phi \wedge \star \gamma-\gamma \wedge \star d \Phi-\delta \gamma \wedge \star \Phi+\Phi \wedge \star d \gamma)(x) .
\end{aligned}
$$

Equations (68) and (69) together are a generalization of Green's identity applicable to inhomogenous differential forms.

\subsection{An integral representation of the fluid velocity}

Equations (34) and (35) lead trivially to

$$
\triangle \tilde{V}_{(j)}=0 .
$$

Therefore using (68), (69), (34) and (35) we find that

$$
\Psi_{(j)}(x)=\left\{\begin{array}{lll}
0 & : & x \notin \overline{\mathcal{D}}_{\Omega(j)}, \\
\frac{1}{2} \tilde{V}_{(j)}(x) & : & x \in b \mathcal{D}_{\Omega(j)}, \\
\tilde{V}_{(j)}(x) & : & x \in \mathcal{D}_{\Omega(j)}
\end{array}\right.
$$

where, for the 2-chain $\Upsilon_{(j) \varepsilon}$ that describes the region $b \mathcal{D}_{\Omega(j)}-\mathcal{B}_{\varepsilon}$,

$$
\Psi_{(j)}(x) \equiv \lim _{\varepsilon \rightarrow 0} \int_{\Upsilon_{(j) \varepsilon}}\left(\delta \gamma \wedge \star \tilde{V}_{(j)}-\tilde{V}_{(j)} \wedge \star d \gamma\right)(x) .
$$

If we act with $\tilde{V}_{D}$ on a test form $\phi$ we find that

$$
\begin{aligned}
\tilde{V}_{D}[\phi] & =\sum_{j \in I} \int_{\Omega_{(j)}} \tilde{V}_{(j)} \wedge \phi \\
& =\sum_{j \in I} \int_{\Omega_{(j)}} \Psi_{(j)} \wedge \phi \\
& =\sum_{j \in I} \int_{\mathcal{M}} \Psi_{(j)} \wedge \phi
\end{aligned}
$$


using (71) and observing that $b \mathcal{D}_{\Omega(j)}$ is a set of measure zero for each $j \in I$. Thus,

$$
\tilde{V}_{D}[\phi]=\int_{\mathcal{M}}\left(\sum_{j \in I} \lim _{\varepsilon \rightarrow 0} \int_{\Upsilon_{(j) \varepsilon}}\left(\delta \gamma \wedge \star \tilde{V}_{(j)}-\tilde{V}_{(j)} \wedge \star d \gamma\right)\right) \wedge \phi .
$$

We now identify pairs of the component 2-cubes of $\left\{\Upsilon_{(1) \varepsilon}, \ldots, \Upsilon_{(N) \varepsilon}\right\}$ as we did earlier for $\left\{\partial \Omega_{(1)}, \ldots, \partial \Omega_{(N)}\right\}$. If $\left\{\sigma_{(k) \varepsilon}^{+}, \sigma_{(k) \varepsilon}^{-}\right\}$is such a pair then we introduce $\Sigma_{(k) \varepsilon}$ via

$$
\begin{aligned}
\Sigma_{(k) \varepsilon}^{*} \alpha & =-\sigma_{(k) \varepsilon}^{+*} \alpha \\
& =\sigma_{(k) \varepsilon}^{-*} \alpha
\end{aligned}
$$

for any $C^{\infty}$ form $\alpha$. Then,

$$
\begin{aligned}
\tilde{V}_{D}[\phi]= & -\int_{\mathcal{M}}\left(\sum_{k \in I_{v}} \lim _{\varepsilon \rightarrow 0} \int_{\Sigma_{(k) \varepsilon}}\left(\delta \gamma \wedge[\star \tilde{V}]_{(k)}-[\tilde{V}]_{(k)} \wedge \star d \gamma\right)\right) \wedge \phi \\
& +\int_{\mathcal{M}}\left(\int_{\partial \Omega}\left(\delta \gamma \wedge \star \tilde{V}_{b}-\tilde{V}_{b} \wedge \star d \gamma\right)\right) \wedge \phi .
\end{aligned}
$$

Applying the junction condition (51) and the boundary condition (52) gives

$$
\begin{aligned}
\tilde{V}_{D}[\phi]= & \int_{\mathcal{M}}\left(\sum_{k \in I_{v}} \lim _{\varepsilon \rightarrow 0} \int_{\Sigma_{(k) \varepsilon}}[\tilde{V}]_{(k)} \wedge \star d \gamma\right) \wedge \phi \\
& -\int_{\mathcal{M}}\left(\int_{\partial \Omega} \tilde{V}_{b} \wedge \star d \gamma\right) \wedge \phi .
\end{aligned}
$$

Therefore, it is natural to introduce a piecewise fluid velocity vector $V$

$$
\tilde{V}(x) \equiv\left\{\begin{array}{lll}
\sum_{k \in I_{v}} \lim _{\varepsilon \rightarrow 0} \int_{\Sigma_{(k) \varepsilon}}[\tilde{V}]_{(k)} \wedge \star d \gamma(x)-\int_{\partial \Omega} \tilde{V}_{b} \wedge \star d \gamma(x) & : & x \in \mathcal{D}_{\Omega} \\
0 & : & x \notin \overline{\mathcal{D}}_{\Omega}
\end{array}\right.
$$

on $\mathcal{M}$, which satisfies

$$
\tilde{V}_{D}[\phi]=\int_{\mathcal{M}} \tilde{V} \wedge \phi
$$


Once the discontinuities in the fluid velocity and its values on the physical boundaries are specified, the velocity of any point $x \in \mathcal{D}_{\Omega}$ can be calculated via (78). Finally, referring back to (71), we note that at any point $x \in \mathcal{D}_{\Sigma(k)}$

$$
V(x)=\frac{1}{2}\left(\lim _{x^{+} \rightarrow x} V_{(k)}^{+}\left(x^{+}\right)+\lim _{x^{-} \rightarrow x} V_{(k)}^{-}\left(x^{-}\right)\right)
$$

where $\left\{V_{(k)}^{+}, V_{(k)}^{-}\right\}$are the smooth fluid velocities on either side of $\mathcal{D}_{\Sigma(k)}$. Equations (78) and (80) are intrinsic versions of well-known expressions used to model the dynamics of vortex sheets (see, for example, $[1,3,7,10]$ ). Note that the limit in (78) can be taken before evaluating the integral if $x \notin \mathcal{U}$ where

$$
\mathcal{U}=\bigcup_{k \in I_{v}} \mathcal{D}_{\Sigma(k)}
$$

\section{Conservation of vorticity}

Let $U$ be a smooth vector field on $\mathcal{D}_{\Omega}$ that satisfies $U(x)=V(x)$ at each $x \in \mathcal{U}$. The $U$-convective derivative on $C^{\infty}$ forms $\alpha$ on $\mathcal{M}$

$$
\dot{\alpha} \equiv \partial_{t} \alpha+\mathcal{L}_{U} \alpha
$$

induces, using (32) and (33), the relation

$$
\dot{T}_{D}[\phi]=\frac{d}{d t}\left(T_{D}[\phi]\right)-T_{D}[\dot{\phi}]
$$

on currents in $\mathcal{M}$. For a chain $c$ to have velocity $U$ means that

$$
\frac{d}{d t} \int_{c} \alpha=\int_{c} \dot{\alpha}
$$

and so, for a current $c_{D}$ to have velocity $U$, where

$$
c_{D}[\phi]=\int_{c} \phi
$$

means that

$$
\dot{c}_{D}=0 \text {. }
$$

Up to now we have considered currents over test forms with compact support on $\mathcal{M}$. For the purposes of this subsection only we restrict ourselves to 
test forms with compact support on $\mathcal{D}_{\Omega}$ in order to focus on the vortex sheets rather than the properties of the flow at the solid boundary. We now demonstrate that vorticity is conserved in the sense that

$$
\partial_{t} \omega_{D}+\mathcal{L}_{U} \omega_{D}=0
$$

over test forms with compact support on $\mathcal{D}_{\Omega}$ if the pressure is continuous across each sheet. The flow on either side of each sheet is composed of the same fluid, and so the surface tension at each sheet should be zero. This also implies that the pressure is continuous (see, for example, page 513 of $[1])$.

The Euler equations are satisfied in each $\mathcal{D}_{\Omega(j)}$,

$$
\partial_{t} \tilde{V}_{(j)}+\nabla_{V_{(j)}} \tilde{V}_{(j)}=-d p_{(j)},
$$

where $p_{(j)}$ is the fluid pressure on $\mathcal{D}_{\Omega(j)}$, and so using

$$
\mathcal{L}_{X} \tilde{X}=\nabla_{X} \tilde{X}+\frac{1}{2} d|X|^{2}
$$

for any smooth vector field $X$ one can show that

$$
\partial_{t} \tilde{V}_{(j)}+\mathcal{L}_{V_{(j)}} \tilde{V}_{(j)}=-d P_{(j)}
$$

where

$$
P_{(j)} \equiv p_{(j)}-\frac{1}{2}\left|V_{(j)}\right|^{2} .
$$

If $\phi$ is a test form with compact support on $\mathcal{D}_{\Omega}$ then

$$
\begin{aligned}
\omega_{D}[\phi] & =d \tilde{V}_{D}[\phi], \\
& =\tilde{V}_{D}[d \phi], \\
& =\sum_{j \in I} \int_{\Omega_{(j)}} \tilde{V}_{(j)} \wedge d \phi .
\end{aligned}
$$

So far we have not said anything regarding the $t$-dependence of $\left\{\Omega_{(1)}, \ldots, \Omega_{(N)}\right\}$. Let us choose them to have velocity $U$. Using (83) and (92),

$$
\frac{d}{d t}\left(\omega_{D}[\phi]\right)=\sum_{j \in I} \int_{\Omega_{(j)}} \dot{\tilde{V}}_{(j)} \wedge d \phi+\omega_{D}[\dot{\phi}],
$$


and so

$$
\dot{\omega}_{D}[\phi]=\sum_{j \in I} \int_{\Omega_{(j)}} \dot{\tilde{V}}_{(j)} \wedge d \phi .
$$

By applying (90) and (91) to (94) one can show that

$$
\dot{\omega}_{D}[\phi]=\sum_{j \in I} \int_{\partial \Omega_{(j)}} f_{(j)} \wedge d \phi
$$

where

$$
f_{(j)} \equiv-p_{(j)}+\iota_{U} \tilde{V}_{(j)}-\frac{1}{2}\left|V_{(j)}\right|^{2} .
$$

As before, let us write (95) in terms of the vortex sheet 2-cubes:

$$
\dot{\omega}_{D}[\phi]=-\sum_{k \in I_{v}} \int_{\Sigma_{(k)}}[f]_{(k)} \wedge d \phi .
$$

However, since

$$
U(x)=\frac{1}{2}\left(\lim _{x^{+} \rightarrow x} V_{(k)}^{+}\left(x^{+}\right)+\lim _{x^{-} \rightarrow x} V_{(k)}^{-}\left(x^{-}\right)\right)
$$

for $x \in \mathcal{D}_{\Sigma(k)}$ one can show that

$$
[f]_{(k)}=-[p]_{(k)}
$$

Hence, if the pressure is continuous across each sheet,

$$
\dot{\omega}_{D}=0
$$

over test forms with compact support in $\mathcal{D}_{\Omega}$.

\section{Self-induced velocity of a single vortex sheet}

Using (78) the self-induced velocity $U$ of a single closed vortex sheet, modelled by the 2-cycle $\Sigma$, in an unbounded flow is

$$
\tilde{U}(x)=\lim _{\varepsilon \rightarrow \infty} \int_{\Sigma_{\varepsilon}}[\tilde{V}] \wedge \star d \gamma(x)
$$


at $x \in \mathcal{D}_{\Sigma}$. Let the vector field $N$ be the outward-pointing normal to $\mathcal{D}_{\Sigma}$. The integral curves of the vector field $\kappa$,

$$
\tilde{\kappa} \equiv \star(\tilde{N} \wedge[\tilde{V}])
$$

are known as sheet vortex lines [10]. It may be prudent, when evaluating (101), to work in a coordinate chart adapted to the sheet vortex lines. The vorticity 2 -current $\omega_{D}$ of $V_{D}$ is

$$
\omega_{D}=\Sigma_{D} \wedge[\tilde{V}]
$$

and since both $\omega_{D}$ and $\Sigma_{D}$ are closed

$$
\begin{aligned}
d \omega_{D} & =0 \\
& =-\Sigma_{D} \wedge d[\tilde{V}] \\
& =-\Sigma_{D} \wedge \underline{d}[\tilde{V}]
\end{aligned}
$$

we see that

$$
\underline{d}[\tilde{V}]=0
$$

where $\underline{d}$ is the exterior derivative on forms on $\mathcal{D}_{\Sigma}$. Locally $[\tilde{V}]=\underline{d} \Gamma$ where, using (102), $\Gamma$ is constant along each sheet vortex line. Moreover, using (100) it can be shown that $\Gamma$ can be chosen to be $t$-independent. Therefore, a natural choice of coordinate chart on the sheet for all $t$ involves assigning a unique value of $\Gamma$ to each sheet vortex $\operatorname{line}^{2}$. Let us assume that the geometry of $\Sigma$ permits this choice at a global level.

For practical calculations, such as those involved in the modelling of the fluids flows present during vortex-induced vibration (see, for example, [2]) of a slender elastic structure, one expects the Frenet curvature of the sheet vortex lines to be much less than the sheet curvature in the $\kappa$-orthogonal direction. The key idea in the evaluation of (101) is to treat the integrations over $\Gamma$ and the sheet vortex lines differently. The sheet is sliced up into $n$ regions each of whose boundaries are described by sheet vortex lines. The slices are replaced by a set of $n$ curves $\left\{C_{(1)}, \ldots, C_{(n)}\right\}$, called representatives, that are themselves sheet vortex lines. The dynamics of the representatives

\footnotetext{
${ }^{2}$ For a curve of vorticity in 2-dimensions this approach leads to the Birkhoff-Rott expression [10] for the vortex curve velocity.
} 
is dictated by an approximate evaluation of (101) over each slice. It is assumed that the width $w_{(j)}(x)$ of the $j$ th sheet slice,

$$
w_{(j)}(x) \equiv \frac{\Delta \Gamma_{(j)}}{|\kappa|}(x),
$$

where $\Delta \Gamma_{(j)}$ is the strength of the $j$ th slice, is much less than the radius of curvature of $C_{(j)}$ at $x \in \mathcal{D}_{C(j)}$. For $w_{(j)}(x) \kappa_{(j)}^{F}(x) \ll 1$ one obtains

$$
\begin{aligned}
\tilde{U}_{(j)}(x) \approx & -\sum_{k \neq j}\left(\frac{\Delta \Gamma}{2 \pi} \frac{\star(\tilde{T} \wedge \tilde{X})}{|\tilde{X}|^{2}}\right)_{(k)}(x) \\
& -\frac{1}{4 \pi}\left(\Delta \Gamma \ln \left(w \kappa^{F}\right) \kappa^{F} \tilde{B}\right)_{(j)}(x)
\end{aligned}
$$

at the point $x \in \mathcal{D}_{C(j)}$. Note that the objects in each term of (107) depend on the point $x \in \mathcal{D}_{C(j)}$ at which $U$ is required and also on a representative (which may or may not be the $j$ th representative). The representative dependence is indicated by a subscript. The vector field $T_{(k)}(x)$ is the normalized tangent to $C_{(k)}(y)$ where $y \in \mathcal{D}_{C(k)}$ is the point of closest approach to $x$. The Frenet curvature and binormal of $C_{(j)}$ at $x \in \mathcal{D}_{C(j)}$ are $\kappa_{(j)}^{F}(x)$ and $B_{(j)}(x)$ respectively.

The contribution of each representative $C_{(k)}, k \neq j$ to the velocity of $C_{(j)}$ at $x \in \mathcal{D}_{C(j)}$ is identical to that given by a rectilinear line vortex of strength $\Delta \Gamma_{(k)}$ aligned along the tangent to $C_{(k)}$ at the point of closest approach to $x \in \mathcal{D}_{C(j)}$. The self-induced contribution to the velocity is of the same form as that obtained for a vortex filament with an $O\left(w_{(j)}(x)\right)$ radius, $1 / \kappa_{(j)}^{F}(x)$ local radius of curvature and strength $\Delta \Gamma_{(j)}$ in the Localised Induction Approximation (LIA), first discovered by Da Rios and Levi-Civita near the beginning of the twentieth century. It is interesting to note that their work remained almost entirely unknown to the rest of the applied mathematics community until more recent times. During the latter half of the twentieth century the LIA has been independently rediscovered by a number of authors (see [8] and [9] for the history, and the mathematics, of this discovery.)

A very similar equation to (107) has been shown by Klein, Majda and Damodaran [4] to hold for the dynamics of arbitrarily long and nearly parallel vortex filaments. 


\section{Conclusion}

The geometry and dynamics of vortex sheets in 3 dimensions have been examined from an intrinsic viewpoint. The velocity of a set of vortex sheets has been derived using two basic ingredients. The first is a generalization of Green's identity for the Poisson-Beltrami equation. The second is a junction condition expressing the continuity of the normal component of the fluid velocity across the sheets. The latter was obtained by representing the fluid velocity as a de Rham current. Conservation of the vorticity 2-current followed from the continuity of pressure across the vortex sheets. A brief summary indicating the route to an approximate expression of the selfinduced velocity of an isolated vortex sheet was given. Its relevance to the classical LIA of vortex filaments was discussed. A detailed presentation of the calculation leading to (107) will be given elsewhere.

\section{Acknowledgments}

We thank our industrial collaborators, Orcina Ltd., for their support. This project is funded jointly by EPSRC, the TCD and Orcina Ltd.

\section{References}

[1] Batchelor, G.K. Introduction to Fluid Dynamics. Cambridge University Press, 1967.

[2] Blevins, R.D. Flow Induced Vibration. Van Nostrand Reinhold, New York, 1990.

[3] Cottet, G-H. and Koumoutsakos, P.D. Vortex Methods : Theory and Practice. Cambridge University Press, 2000.

[4] Klein, R., Majda, A.J. and Damodaran, K. Simplified equations for the interaction of nearly linear parallel vortex filaments. J. Fluid Mech. 288, 1995, 201-248.

[5] Nickerson, H.K., Spencer, D.C. and Steenrod, N.E. Advanced Calculus. New York : Van Nostrand, 1959.

[6] de Rham, G. Differentiable Manifolds. Springer-Verlag, 1984.

[7] Pozrikidis, C. Introduction to Theoretical and Computational Fluid Dynamics. Oxford University Press, 1997.

[8] Ricca, R.L. Rediscovery of Da Rios equations. Nature 352, 1991, 561-562. 
[9] Ricca, R.L. The contributions of Da Rios and Levi-Civita to asymptotic potential theory and vortex filament dynamics. Fluid Dynamics Research 18,1996,245-268.

[10] Saffman, P.G. Vortex Dynamics. Cambridge University Press, 1992.

\section{Geometrija i dinamika trodimenzionalnih vrtložnih površi}

UDK $514.7,532.59$

Posmatramo osobine i dinamiku vrtložnih površi sa geometrijskog stanovišta bez uvodjenja koordinata. Forme sa distribucionim vrednostima (de Rhamove struje) se koriste za reprezentaciju brzine i vrtložnosti fluida koje potiču od vrtložnih površi. Glatke brzine na obe strane ovih površi su rešene u zavisnosti od jačine površi korišćenjem jezika dvostrukih formi. Na ovaj način su izraženi klasični rezultati koji se odnose na neprekidnost komponente brzine u pravcu normale na površ i konzervacija vrtložnosti. Formalizam je tada primenjen na slučaj samo-indukovane brzine neke izolovane vrtložne površi. Razvijen je uprošćeni izraz za brzinu površi preko reprezentativnih krivih. Njegova veza sa klasičnom aproksimacijom lokalizovane indukcije (LIA) dinamike vrtložnih niti je diskutovana. 